\title{
Errors in Tooth Preparation for Complete Veneer Crowns
}

\author{
Navya Khanna1, Dhanraj M Ganapathy² and Subhabrata Maiti ${ }^{3}$ \\ ${ }^{1}$ Saveetha Dental College and Hospitals, Saveetha Institute of Medical and \\ Technical Sciences (SIMATS),Saveetha University, Chennai- 77, India. \\ ${ }^{2}$ Professor and head, Department of Prosthodontics, Saveetha Dental College and \\ Hospitals, Saveetha Institute of Medical and Technical Sciences (SIMATS), Saveetha \\ University, Chennai-77, India. \\ ${ }^{3}$ Senior Lecturer, Department of Prosthodontics, Saveetha Dental College and \\ Hospitals, Saveetha Institute of Medical and Technical Sciences (SIMATS), Saveetha \\ University, Chennai-77, India.
}

\section{ABSTRACT}

Full-crown restoration is a common accomplishment in restorative dentistry and some dentists believe that so many crowns are mounted instead of more modest restorations. The explanations for the widespread use of full crowns are clear. Third-party billing agencies usually routinely bill for full crowns, but frequently refuse payment for more limited restorations such as inlays and onlays, or pay the premium for amalgam restoration. Whatever the causes, the hallmark of teeth-restorative dentists that are mostly carious or otherwise worn down is full-crown reconstruction. Many problems in tooth preparations for full crowns emerge immediately in any conversation with dental laboratory owners and working laboratory technicians regarding the adequacy of tooth preparations. As observed in dental laboratories, crown preparations for teeth often have numerous shortcomings which limit the ability of laboratory technicians to provide their dental clients with optimum service. The aim of the study is to find the errors in tooth preparation, categorise it and study their prevalence.

\section{KEY WORDS: ERRORS,TOOTH PREPARATION,CROWNS.}

\section{INTRODUCTION}

The form of prepared teeth and the amount of tooth structure removed are important contributors to the mechanical, biologic, and aesthetic success of the overlying crown or fixed partial denture. (Ozdemir et

\section{ARTICLE INFORMATION}

*Corresponding Author: subhabratamaiti.sdc@saveetha.com Received 4th Aug 2020 Accepted after revision 29th Sep 2020 Print ISSN: 0974-6455 Online ISSN: 2321-4007 CODEN: BBRCBA

Thomson Reuters ISI Web of Science Clarivate Analytics USA and Crossref Indexed Journal

\section{Clarivate crossef}

NAAS Journal Score 2020 (4.31) SJIF: 2020 (7.728)

A Society of Science and Nature Publication,

Bhopal India 2020. All rights reserved.

Online Contents Available at: http//www.bbrc.in/

Doi: http://dx.doi.org/10.21786/bbrc/13.8/188 al., 2007) Therefore, it is important to develop clinical guidelines that can be used to optimise success in fixed prosthodontics. Errors in following these guidelines will lead to compromise in the longevity and placement of these prosthesis.(Ayad, Maghrabi and Rosenstiel, 2005) (Jain and Dhanraj, 2016).

Occlusal reduction, axial reduction, taper, finishing line, and undercuts in axial walls influence the consistency of planning for extra coronal restorations. Goodacre et al. reviewed medical preparedness recommendations and recommended nine science standards to guide the preparation(Goodacre, Campagni and Aquilino, 2001). This has been proposed to be 10-20 degrees as an optimal minimum angle of occlusal convergence. Suggested to be $3 \mathrm{~mm}$ for incisors and premolars and $4 \mathrm{~mm}$ for

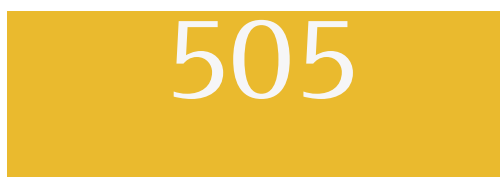


molars is the minimal occluso-cervical dimension for 10 percent-20 per occlusal convergence. For all metals, $1 \mathrm{~mm}$ and $2 \mathrm{~mm}$ respectively, for metal ceramics and for all semi-translucent ceramic processes, axial and occlusal cuts are $0.5 \mathrm{~mm}$ to $1 \mathrm{~mm}$ long. The shape of the prepared teeth and the volume of tooth tissue removed are significant contributors to mechanical, biological, and cosmetic efficiency of the overlying crown or fixed partial dentures.

Therefore it is important to develop clinical guidelines which can be used to improve performance in fixed prosthodontics. Errors in following these instructions can influence the durability of these prostheses and their positioning. The quality of the planning for extra coronal restorations is influenced by occlusal reductions, axial contraction, taper, finish line and undercuts in axial walls. Goodacre et al provided instructions for the clinical preparation and suggested nine basic principles to guide the preparation. It has been proposed that the mean occluso cervical scale is $3 \mathrm{~mm}$ for incisors and premolars, and $4 \mathrm{~mm}$ for molars.

The axial and occlusal cuts are $0.5 \mathrm{~mm}$ and $1 \mathrm{~mm}$ deep in all metals, $1 \mathrm{~mm}$ and $2 \mathrm{~mm}$ respectively, in metal ceramics and all semi-translucent ceramic systems. Given the stringent standards set for teeth training, laboratory technicians are still facing difficulties in this area. Errors in tooth preparation were established as a second laboratory problem immediately upon insufficient impression. The occurrence of dental planning issues over a long period of time. Such skills may be difficult to obtain, or there may be a lack of credit hours to teach this subject, or there may be no direct communication between the dentist and the laboratory technician. The problem has been the area of focus for several investigations which evaluated tooth preparation to see how much it is far from the ideal.(Christensen, 2007a) (Christensen, 2007b)

Failure in tooth preparation was identified as a second laboratory challenge immediately after impression was inadequate(Buchanan, 2000). The persistence of the problems of tooth preparation over a long period of time is remarkable and the occurrence of the same defects is doubtful given the documents. It may be that these skills are difficult to learn, or there is a shortage of credit hours in teaching this subject, or there may be no clear contact between the dentist and the technician in the laboratory. This question was the focus of many investigations which assessed the preparation of the tooth to see how far it is from the ideal.

These studies targeted preparations done by dental students, teaching staff, general practitioners, residents and prosthodontists, and different results have been reported. Most previous studies focused on one aspect of tooth preparations mainly taper, and few studies incorporated other aspects of tooth preparation in addition to taper. The study targeted preparation done by dental students(Mack, 1980; Noonan and Goldfogel, 1991; Dorriz et al., 2008; Selvan and Ganapathy, 2016), teaching staff(Patel, Wildgoose and Winstanley, 2005), general practitioners(Enechi, 2004), residents(Nordlander et al., 1988) and prosthodontics(Arnetzl and Dornhofer, 2004) and different results have been reported. Most previous studies focused on one aspect, ie- taper, and a few studies incorporated other aspects of tooth preparation, in addition to taper(Sato et al., 1998).

Most of these were carried out in Europe, North America and Asia. Very little information is available concerning the ability of dentists in India to prepare teeth for full veneer crowns. There are many studies which followed the performance of dental students in tooth preparation for fixed prosthodontics during their pre-clinical course and efforts for accurate evaluation of their preclinical work were done. The aim of this study is to evaluate the quality of tooth preparation for complete veneer casting restorations performed by dental students in their Chennai, India.

\section{MATERIAL AND METHODS}

The study setting was a clinical setting which involved casts of patients sent to dental technicians for fabrication. The approval of SRB of Saveetha Dental College and patient approval to use their casts was seeked. The number of people involved in the study was one, ie- the analyser. This was an in vitro study and proper sampling was done to minimalism sampling bias. The sampling method was a convenient sampling technique from a trimmed stone model. The number of casts studied was 100. This, although, was a pilot study and further studies will larger sample size and more quantitative will be taken. At the end, the data was verified by the Department of Prosthodontics. The inclusion criteria was single unit crowns, and the exclusion criteria was multiple missing, or fixed partial dentures.

\section{RESULTS}

Tooth preparation is one of the key reasons affecting a restaurant's sustainability. A lot of facets of tooth preparation were analyzed in the present report. In this research, greater care was taken as described in the section on materials and methods to ensure adequate seating of the analysis casts impression on the working casts to remove or reduce any inaccuracies in the seating. We have tried to identify and discuss the major challenges into preparation noted by laboratory technicians and confirmed by dentists.

Figure 1 shows that reduction of about 44\% of the casts under study were done correctly, whereas 32\% were overreduced. A minority of $24 \%$ casts were under reduced. Figure 2 illustrates the iatrogenic damage caused to the adjacent tooth due to incorrect method of tooth preparation. A little above half the samples (56\%) showed no iatrogenic damage, whereas in the remaining 44\% iatrogenic damage was present. Figure 3 throws light on the definition of finish lines of the tooth preparation. A majority of 66\% were well defined finish lines, and only $34 \%$ were not well defined. 
Figure 1 : Reduction of about $44 \%$ of the casts under study were done correctly, whereas $32 \%$ were over-reduced. A minority of $24 \%$ casts were under reduced.

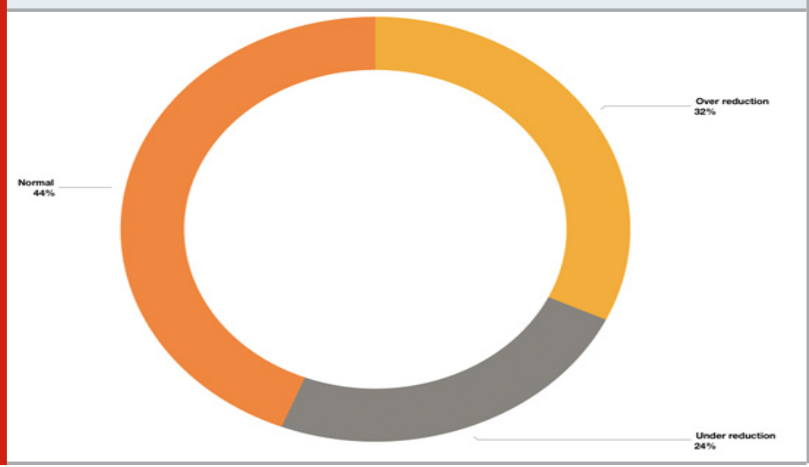

Figure 2 : Iiatrogenic damage caused to the adjacent tooth due to incorrect method of tooth preparation. 56\% showed no iatrogenic damage, whereas in the remaining $44 \%$ iatrogenic damage was present.

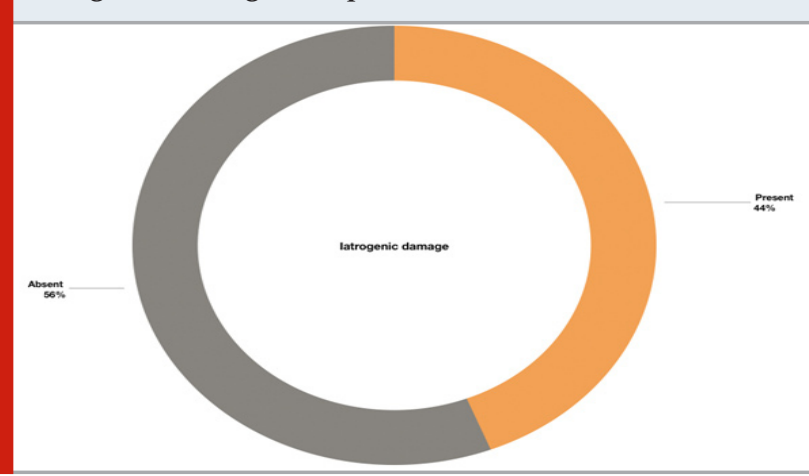

Figure 3 : Definition of finish lines of the tooth preparation. A majority of $66 \%$ were well defined finish lines, and only $34 \%$ were not well defined.

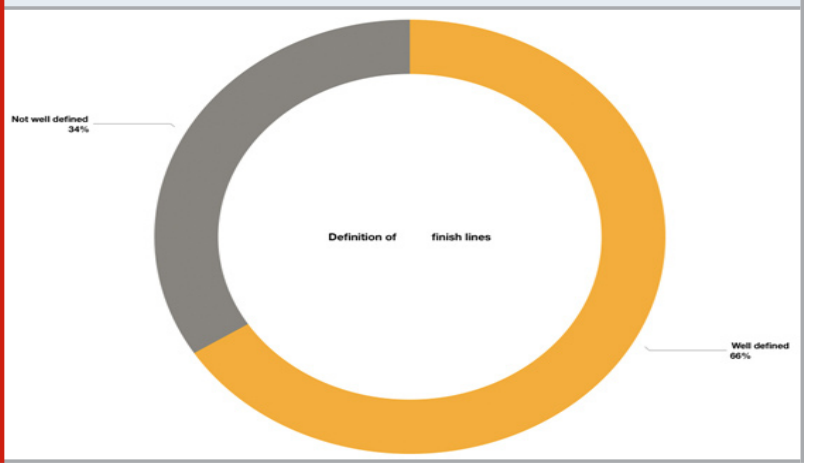

Figure 4 evaluates the taper, and 3/5ths of the casts (60\%) have proper taper, but in 40\% taper is absent. Figure 5 shows that a little over half, 51\% have irregular tooth surfaces after tooth preparation, whereas 49\% have regular surfaces. Figure 6 illustrates that there are equal number of casts with presence and absence of functional cuspal bevel, ie- 50\% each. Figure 7 demonstrates that $52 \%$ of the dies had secondary retention features present, whereas in $48 \%$ they were absent. Figure 8 states that a majority of $68 \%$ had undercuts absent, and only $32 \%$ had unwanted undercuts present. In figure 9, it is illustrated that a fair majority of $66 \%$ of the casts followed the correct path of insertion, whereas 34\% had an incorrect path of insertion.

Figure $4: 60 \%$ of the casts have proper taper, but in $40 \%$ taper is absent.

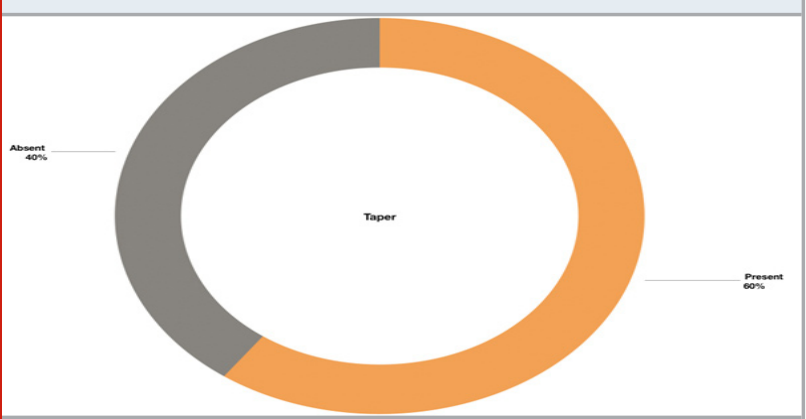

Figure 5: 51\% have irregular tooth surfaces after tooth preparation, whereas $49 \%$ have regular surfaces.

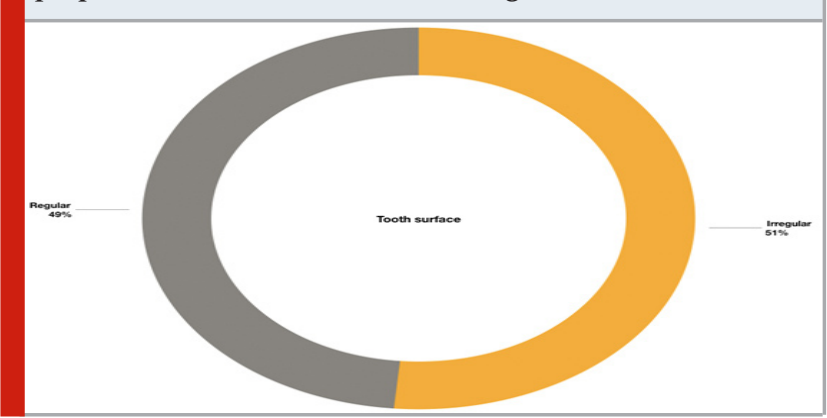

Figure 6: Equal number of casts with presence and absence of functional cuspal bevel, ie- 50\% each.

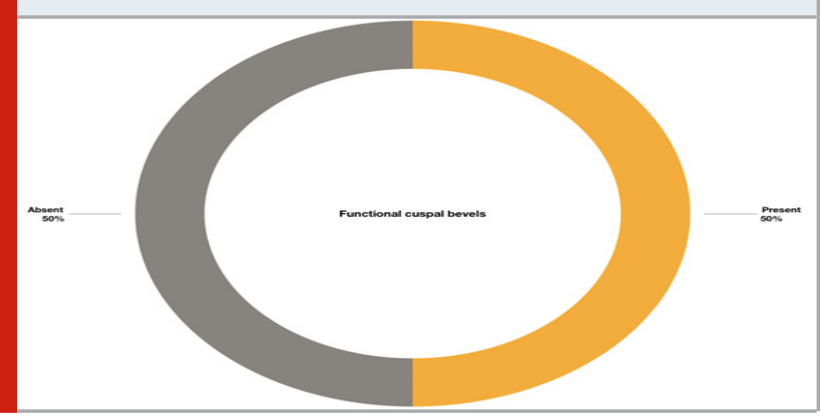

Figure $7: 52 \%$ of the dies had secondary retention features present, whereas in $48 \%$ they were absent.

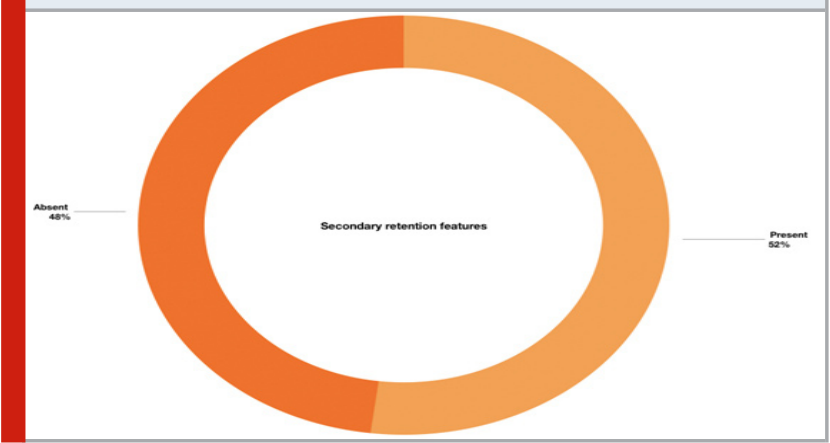


Figure $8: 68 \%$ of the dies had undercuts absent, and only $32 \%$ had unwanted undercuts present.

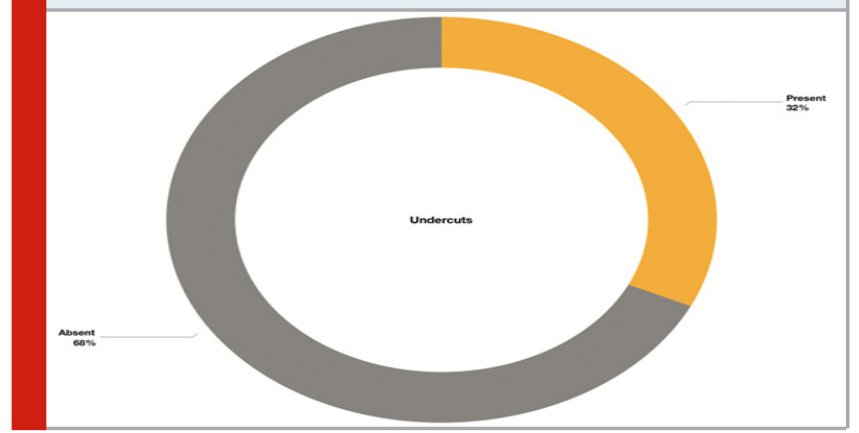

Figure 9: 66\% of the casts followed the correct path of insertion, whereas $34 \%$ had an incorrect path of insertion.

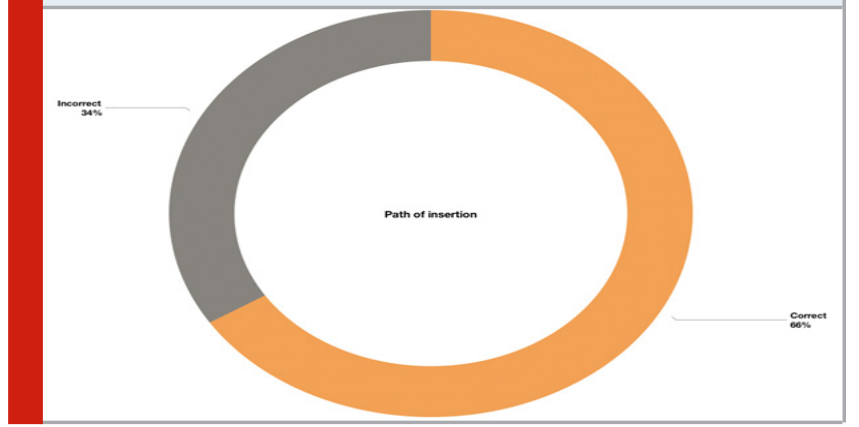

DISCUSSION

Failures not unusual in complete veneer crowns. Unfortunately one or two such failures are sufficient to discourage the clinician from fully using the material. The defects that occur during tooth preparation due to mistakes are solely the dentist 's fault and can be effectively prevented by taking proper treatment. Good working knowledge, good clinical judgment in the selection of cases and execution of the planned treatment will guarantee success. Knowing the material properties to a large degree and keeping up with the latest developments and new creativity would help minimize chairside time and still help us produce consistent results with good care.

Although the students have the concepts of tooth preparation fresh in their mind it appears that the lack of clinical experience is the primary cause of the large taper in their preparations as most of the preparations in the present study were their first clinical case, so getting a large taper may not be surprising. Some dentists use small, rounded, tapered, or flame-shaped diamonds to reduce the medial, distal, lingual, and even facial walls of crown prepared dentures. It minimizes tooth density and builds feather edge margins. Although this method of preparation is suitable for all metal restorations, it is not appropriate for metal ceramic and all-ceramic crowns, because it leaves inadequate thickness for both substructure and veneering ceramics.
Inadequate axial tooth preparation forces technicians to make over contoured crowns and compromise both the esthetics and self-cleansing design. (Christensen, 2007a) The failure to use such diamond burs could have led to inadequate tooth reductions in the study. This problem could have been eliminated by using adjustable clearance tabs to test for sufficient occlusal minimization.(Adams, 2004). It could also have occured because of the dentist 's inherent fear of exposure to pulp. These fears may be allayed by knowledge of tooth anatomy and sound clinical judgement. Copious spray of water should be used during the process of preparing a tooth. The occlusal reduction for the restoration should have indentations where the original tooth anatomy had indentations. (Smith et al., 1999).

Finish line should be well defined, that is, no sharp line or point angle should be present. Leaving behind sharp line angles and point angles in the preparation can result in major fit issues and time-consuming appointments at the cementation time. For finishing margins diamond burs are not recommended. Also the super-fine burs create a rough finish. The surface roughness which they produce is directly proportional to the bur grit grains. Tungsten carbide burs, offering a cleaner range, prove to be stronger in the end. The ideal surface smoothness is produced by a tungsten carbide finish bur $(20,000$ rpm) Failure to use the required armamentarium cloud have led to sharp line angles from possible sites for all ceramic(Ganapathy and Sathyamoorthy, 2016) restoration which lead to fracture stimulation.

The solution to this is to round all of the straight line and point angles at the time of preparation. Bevelling of margins or creating a feather edge margin is contra indicated for all-ceramic preparation. To fabricate and all-ceramic restoration over bevelled finish lines is nearly impossible(Kissov and Chalashkanova, 2001). For pressable ceramics(Ranganathan, Ganapathy and Jain, 2017), shoulder is required for strength at the margins because marginal areas bear much more support of the crown in function. This is because the best adaptation of the crown is at the cervical aspect(Kiliçarslan et al., 2004). For all ceramic sure, chamfer is preferred as the metal used, that is alumina or zirconia are inherently strong and do not require bulk of material for strength as is true for conventional ceramics.

The main reason for deciding on this methodology was to get a wide overview of all the aspects of tooth preparation at once. We could then analyze as to which parameter deviated at most. The advantage of this study lies in its wider spectrum of parameters. In a study by Noonan (Noonan and Goldfogel, 1991), the convergence angle was found to be related ro the taper of the cast. And most of the casts had accurate taper, which is in keeping with our results. The degree of preparedness taper has long been the focus of controversy. Until the invention of resin cements, the dentist had to rely in large measure on a strong retentive method to hold the crown in place. Today the situation has changed for the 
resin cements. It is no longer considered essential to reach near parallel surfaces, as these cements bond very tightly to the surface of the tooth. But the taper of the prepared tooth from the long axis of the tooth should not exceed 8 or 10 degrees. By using cements which do not bind to the tooth surface, it seems appropriate to make the arrangements more parallel. (Goodacre, 2004)

The degree of taper to prepare the tooth has long been a debatable subject. Before the invention of resin cements , the dentists had to rely primarily on good retentive form to hold the crown in place(Prakash, Ganapathy and Mallikarjuna, 2019). The scenario is different today, with resin cement. It is no longer considered critical to achieve a near parallel surface, since the cements bond very well to the tooth surface (Leles and Compagnoni, 2001). Investigating a die has verified that it is very difficult in practice to get theoretical values of the convergence angle(Leempoel et al., 1987). Studies on the students' taper found that mean taper ranges ranged from $11^{\circ}$ to $27^{\circ}$. The mean taper of clinical anteriors(Jain et al., 2018) was $31.6^{\circ}$ buccolingually and $16.8^{\circ}$ mesiodistally, and the clinical posteriors was $16.8^{\circ}$ buccolingually and $22.4^{\circ}$ mesiodistally(Rafeek et al., 2006).

The presence of undesired undercuts is frequently observed due to complicating clinical factors such as poor visibility and access. It hinders the entire cast restoration position, which may result in the need to recontour the prepared tooth to create a new impression. If such undercuts are left unattended, they must be blocked by laboratory technicians before the reconstruction is finished. We contribute to bad internal component match and potentially decrease restore retention (Marghalani, 2016). Retentive grooves, undercuts, channels, and retentive pins are used to increase the bond to dentin that bonding agents produce. In a study by The main limitation of this study was that it was a pilot study carried out for an overview of understanding this topic. The errors were studied qualitatively, instead of quantitatively. Thus, the accuracy might not be at par with what metric analysis could have given. Visual estimation errors might have led to inaccuracy in the results.

Thus, tooth preparation is one of the main factors that influence the longevity of the restoration. The quality of preparation for extracoronal restorations is affected by occlusal reduction, axial reduction, taper, finishing line and undercuts in axial walls. Incorrect tooth preparation can severely compromise the retention, aesthetics, strength and service potential of subsequently placed crowns. Thus, more rigorous awareness and training programmes need to be initiated to address this.

The success of a fixed prosthodontic treatment can be determined by disciplined preparation of the tooth. This can fix, replace and adjust the dentition components to enhance the function, esthetics and health of the hard and soft tissues. Under this study's constraint, it may be argued that the taper the dental students obtained was greater than was suggested in the dental literature. Given the rise in taper there were no new improvements to enhance retention. As contrasted with the standard, occlusal and axial reductions were considered appropriate. While discontinuity was not unusual, most arrangements had straight and consistent finishing lines. Preparations were majorly smooth, with less irregularities or sharp angles.

A mental image of the design of the artificial crown and the anticipated occlusion must precede the reduction in the structure of the dent. In addition to mathematical principles related to the preparation of the tooth, the operator will consider the importance of the burs, the patient's location and his own location, the rests of the hand and finger and the level of reduction in order to ensure a good preparation of the tooth.In future, this study can be carried out with a larger sample size. It can include and compare full veneers with multiple missing units and fixed partial denture tooth preparations. The study can also compare the errors individually among each year of undergraduate students.

\section{CONCLUSION}

The main recommendations of this study were to increase the duration of the preclinical course, and the clinical practice of tooth preparation should be given more emphasis. Long-term clinical studies are required to fully assess the longevity of complete veneered restorations in order to see the less than ideal taper influence on the success of these restorations.

\section{ACKNOWLEDGEMENTS}

The authors are thankful to Saveetha Dental College for providing a platform to express our knowledge.

Conflict of Interest: The authors declare no conflict of interest.

\section{REFERENCES}

Adams, D. C. (2004) 'The ten most common all-ceramic preparation errors: a doctor/technician liaison's perspective', Dentistry today. mabeldental.com, 23(10), pp. 94, 96-9.

Arnetzl, G. and Dornhofer, R. (2004) 'PREPassistant: a system for evaluating tooth preparations', International journal of computerized dentistry. europepmc.org, 7(2), pp. 187-197.

Ayad, M. F., Maghrabi, A. A. and Rosenstiel, S. F. (2005) 'Assessment of convergence angles of tooth preparations for complete crowns among dental students', Journal of dentistry. Elsevier, 33(8), pp. 633-638.

Buchanan, L. S. (2000) 'The standardized-taper root canal preparation-Part 1 . Concepts for variably tapered shaping instruments', International endodontic journal. Wiley Online Library. Available at: https://onlinelibrary. wiley.com/doi/abs/10.1046/j.1365-2591.2000.00384.x. Christensen, G. J. (2007a) 'Frequently encountered errors in tooth preparations for crowns', Journal of the American Dental Association . jada.ada.org, 138(10), 
pp. 1373-1375.

Christensen, G. J. (2007b) 'When is a full-crown restoration indicated?', Journal of the American Dental Association . jada.ada.org, 138(1), pp. 101-103.

Dorriz, H. et al. (2008) 'The convergence angle of fullcoverage crown preparations made by dental students'. JOURNAL OF DENTISTRY OF TEHRAN UNIVERSITY OF MEDICAL SCIENCES. Available at: https://www.sid.ir/ en/Journal/ViewPaper.aspx?ID=122639.

Enechi, T. C. (2004) The taper of clinical crown preparations done by dental students and dentists in two African countries: a comparative analysis. University of the Western Cape. Available at: http://etd.uwc.ac.za/ handle/11394/1546.

Ganapathy, D. and Sathyamoorthy, A. (2016) 'Effect of resin bonded luting agents influencing marginal discrepancy in all ceramic complete veneer crowns', Journal of clinical and. ncbi.nlm.nih.gov. Available at: https://www.ncbi.nlm.nih.gov/pmc/articles/ PMC5296581/.

Goodacre, C. J. (2004) 'Designing tooth preparations for optimal success', Dental clinics of North America. dental.theclinics.com, 48(2), pp. v, 359-85.

Goodacre, C. J., Campagni, W. V. and Aquilino, S. A. (2001) 'Tooth preparations for complete crowns: an art form based on scientific principles', The Journal of prosthetic dentistry. Elsevier, 85(4), pp. 363-376.

Jain, A. R. et al. (2018) 'Determination of correlation of width of maxillary anterior teeth using extraoral and intraoral factors in Indian population: A systematic review', World J Dent. researchgate.net, 9, pp. 68-75. Jain, A. R. and Dhanraj, M. (2016) 'A clinical review of spacer design for conventional complete denture', Early pregnancy: biology and medicine: the official journal of the Society for the Investigation of Early Pregnancy. HATASO Enterprises LLC, 8(5), p. 1.

Kiliçarslan, M. A. et al. (2004) 'In vitro fracture resistance of posterior metal-ceramic and all-ceramic inlay-retained resin-bonded fixed partial dentures', The Journal of prosthetic dentistry. europepmc.org, 92(4), pp. 365-370.

Kissov, H. K. and Chalashkanova, M. I. (2001) 'The impression as a means for analysis of clinical mistakes in fixed prosthodontics', Folia medica. europepmc.org, 43(1-2), pp. 84-87.

Leempoel, P. J. et al. (1987) 'The convergence angle of tooth preparations for complete crowns', The Journal of prosthetic dentistry. thejpd.org, 58(4), pp. 414-416. Leles, C. R. and Compagnoni, M. A. (2001) 'A simple method to detect undercuts during tooth preparation for fixed prosthodontics', The Journal of prosthetic dentistry. fac.ksu.edu.sa, 85(5), pp. 521-522.

Mack, P. J. (1980) 'A theoretical and clinical investigation into the taper achieved on crown and inlay preparations', Journal of oral rehabilitation. Wiley Online Library, 7(3), pp. 255-265.

Marghalani, T. Y. (2016) 'Frequency of undercuts and favorable path of insertion in abutments prepared for fixed dental prostheses by preclinical dental students', The Journal of prosthetic dentistry. Elsevier, 116(4), pp. 564-569.

Noonan, J. E., Jr and Goldfogel, M. H. (1991) 'Convergence of the axial walls of full veneer crown preparations in a dental school environment', The Journal of prosthetic dentistry. Elsevier, 66(5), pp. 706-708.

Nordlander, J. et al. (1988) 'The taper of clinical preparations for fixed prosthodontics', The Journal of prosthetic dentistry. Elsevier, 60(2), pp. 148-151.

Ozdemir, N. et al. (2007) 'An evaluation of the causes and the methods of removal of fixed partial dentures', EÜ Di冈hek Fak Derg, 28, pp. 168-177.

Patel, P. B., Wildgoose, D. G. and Winstanley, R. B. (2005) 'Comparison of convergence angles achieved in posterior teeth prepared for full veneer crowns', The European journal of prosthodontics and restorative dentistry. researchgate.net, 13(3), pp. 100-104.

Prakash, M. S., Ganapathy, D. M. and Mallikarjuna, A. V. (2019) 'Knowledge awareness practice survey on awareness of concentrated growth factor among dentists', Drug Invention Today. search.ebscohost.com, 11(3). Available at: https://bit.ly/36HmUWO

Rafeek, R. N. et al. (2006) 'Abutment taper of full cast crown preparations by dental students in the UWI School of Dentistry', The European journal of prosthodontics and restorative dentistry. europepmc. org, 14(2), pp. 63-66.

Ranganathan, H., Ganapathy, D. M. and Jain, A. R. (2017) 'Cervical and Incisal Marginal Discrepancy in Ceramic Laminate Veneering Materials: A SEM Analysis', Contemporary clinical dentistry. ncbi.nlm. nih.gov, 8(2), pp. 272-278.

Sato, T. et al. (1998) 'A clinical study on abutment taper and height of full cast crown preparations', Journal of medical and dental sciences. jstage.jst.go.jp, 45(3), pp. 205-210.

Selvan, S. R. and Ganapathy, D. (2016) 'Efficacy of fifth generation cephalosporins against methicillin-resistant Staphylococcus aureus-A review', Research Journal of Pharmacy and Technology. A \& V Publications, 9(10), pp. 1815-1818.

Smith, C. T. et al. (1999) 'Effective taper criterion for the full veneer crown preparation in preclinical prosthodontics', Journal of prosthodontics: official journal of the American College of Prosthodontists. Wiley Online Library, 8(3), pp. 196-200. 